\title{
Einfach wirkungsvoll? - Erkenntnisse und Methoden der Wirkungsbeobachtung in der entwicklungspolitischen Inlandsarbeit
}

Der Tagungsort, das Langenbeck-Virchow-Haus mitten im Berliner Klinikviertel war für viele neu, die Fragen zu den Wirkungen der entwicklungspolitischen Inlandsarbeit, die dort im Mittelpunkt standen, hingegen vielen vertraut. 92 Teilnehmende, davon die meisten aus NGOs, aber auch aus Förderinstitutionen, dem BMZ sowie vereinzelt auch Wissenschaftler/innen, waren auf die Ergebnisse des Forschungsvorhabens „Wirkungen und Methoden der Wirkungsbeobachtung in der entwicklungspolitischen Inlandsarbeit" gespannt. Gut zwei Jahre lang hatte das aus fünf Personen bestehende Konsortium, begleitet von einer Steuerungsgruppe mit Vertreter/inne/n von agl, BMZ, Brot für die Welt/Evangelischer Entwicklungsdienst, CARE, Christoffel Blindenmission, Engagement Global, EPIZ Berlin, Kindernothilfe, Misereor, Stiftung Nord-Süd-Brücken sowie der VENRO-Geschäftsstelle, folgende drei Hauptfragen bearbeitet:

1. Welche Wirkungen lassen sich in der entwicklungspolitischen Bildungs- und Informationsarbeit realistischer Weise erwarten?

2. Welche Merkmale lassen sich identifizieren, die die Wirksamkeit von Maßnahmen der entwicklungspolitischen Bildungs- und Informationsarbeit befördern können?

3. Wie lassen sich Wirkungen im Rahmen von Evaluationen in der entwicklungspolitischen Bildungs- und Informationsarbeit effizient erfassen?

Nach der Eröffnung der Tagung durch VENRO Vorstandsmitglied Chris Boppel und Dr. Bernhard Felmberg, Abteilungsleiter Z / Zentralabteilung Zivilgesellschaft und Kirchen im BMZ, begann Eva Quiring vom Konsortium mit einem Überblick über die Forschungsfragen, das Design, den zeitlichen Ablauf der Studie sowie das der Studie zugrundeliegende, umfassende Sample. Dieses wurden in insgesamt acht untersuchten Fallbeispielen sehr unterschiedlicher NGOs erhoben, die sich für die Teilnahme an der Studie beworben hatten, und gemeinsam mit der Steuerungsgruppe aus 24 eingereichten Projekten ausgewählt worden waren. Zwei Fallbeispiele wurden je einem der folgenden Maßnahmentypen zugeordnet, die einen Großteil der in Deutschland typischen Aktivitäten der entwicklungspolitischen Informationsund Bildungsarbeit abbilden : (a) Kurzveranstaltungen, Projekt-/ Seminartag, und Projekt-/Seminarwoche, (b) schulbezogene Kampagnen, (c) Schulung von Multiplikator/inn/en sowie (d) Entwicklung und Einsatz von Materialien. Susanne Höck präsentierte im Anschluss zentrale Ergebnisse der Studie. Die in den Fallbeispielen erhobenen Wirkungen wurden mit Befunden einer umfangreichen Desk-Studie (Grundlage 97 Studien) zusammengeführt und in vier empirisch breit fundierte, auf unterschiedliche Bereiche der entwicklungspolitischen Bildungs- und Informationsarbeit ausgerichtete Wirkungsmodelle gefasst. Diese bilden nicht nur die festgestellten Wirkungen ab, sondern auch die vielfältigen wirkungsrelevantem Einflussfaktoren und Wirkungsbezüge. Nach einem Zwischenfazit fokussierte Bernward Causemann im dritten Teil der Präsentation v.a. auf die methodischen Herausforderungen der Wirkungserhebung, die sich vor allem aus der Komplexität von Lernen, dem zeitlich versetzten Eintritt von Wirkungen sowie der teilweise schwierigen Versprachlichung von Lernwirkungen ergeben, und schloss mit einigen Überlegungen zur weiteren Anregung des Diskurses über die Wirkungserfassung von Bildungsprozessen. Nach einer Diskussion im Plenum standen in drei Arbeitsgruppen am Nachmittag nochmals die Wirkungsmodelle und die darin abgebildeten Wirkungszusammenhänge im Mittelpunkt. Gleichzeitig konnten die Teilnehmenden auch Einblick in die Methodik der Datenerhebung und detaillierte Befunde in den einzelnen Maßnahmentypen gewinnen. In der abschließenden Podiumsdiskussion mit Vertreter/inne/n aus NGOs, Förderinstitutionen und Wissenschaft stand zum einen die Rolle der Wirkungsorientierung für die entwicklungspolitische Bildungsarbeit im Fokus. Die Komplexität von Lernprozessen stellt eine große Herausforderung dar, wenn es darum geht Wirkungen zu erfassen. Dabei wurde unter anderem kontrovers diskutiert, wie dies im Rahmen von geförderten Projekten berücksichtigt werden kann. Zum anderen widmete sich die Diskussion der Frage, wie Wirkungsorientierung in der Praxis handhabbar gemacht werden kann und so wieder ein Instrument des Lernens für die Akteurinnen und Akteure der Bildungsarbeit wird.

Insgesamt hat die Konferenz verdeutlicht, dass die Wirkungsstudie einen wichtigen Meilenstein im Diskurs darstellt, diesen aber nicht beschließt. Der Wunsch der Praxis ist groß, die Qualität und Wirksamkeit der eigenen Arbeit zu verbessern, sodass knappe Ressourcen dort eingesetzt werden können, wo sie Wirkung entfalten. Nun liegt es an den Aktiven, selbst zu diskutieren, wie die Ergebnisse in wirkungsorientierte Arbeitsansätze in der entwicklungspolitischen Bildungsarbeit überführt werden können. Dabei geht es auch um Fragen der Qualität von Bildungsmaßnahmen. Wie können Qualitätskriterien für die entwicklungspolitische Bildungsarbeit vor dem Hintergrund der Ergebnisse verbessert und erweitert werden? VENRO plant deshalb, weiterhin die kritische Reflexion und das gemeinsame Lernen der NGOs mit Angeboten wie Praxisworkshops, Fortbildungen und Wissensbereitstellung zu begleiten. Das gesamte Forschungsvorhaben wird spätestens zu Beginn des Jahres 2019 in der Reihe „Erziehungswissenschaft und Weltgesellschaft" im Waxmann Verlag publiziert und gleichzeitig auch als Open Access verfügbar sein.

Susanne Höck, Mitglied des Konsortiums;

Katharina Stahlecker, VENRO doi.org/10.31244/zep.2018.04.08 